\title{
Aplicación móvil para el seguimiento de los cursos de la plataforma de Ambiente Virtual de Aprendizaje
}

\section{Mobile application to follow the courses of the Virtual Learning Environment Platform}

\author{
RAFAEL-PÉREZ, Eva †*, MORALES-HERNÁNDEZ, Maricela, MIRAMÓN-SANTIAGO, Diana del \\ Carmen y HERNÁNDEZ-ÁBREGO, Anayansi Cristina
}

Instituto Tecnológico de Oaxaca, Departamento de Sistemas y Computación., Avenida Ing. Víctor Bravo Ahuja No. 125 Esquina Calzada Tecnológico, C.P. 68030

ID 1 ${ }^{\text {er }}$ Autor: Eva, Rafael-Pérez / ORC ID: 0000-0003-2793-1254, CVU CONACYT ID: 905268

ID $1^{\text {er }}$ Coautor: Maricela, Morales-Hernández / ORC ID: 0000-0002-3521-2041, CVU CONACYT ID: 731036

ID $2^{\text {do }}$ Coautor: Diana del Carmen, Miramón-Santiago / ORC ID: 0000-0003-4397-9864, CVU CONACYT ID: 999879

ID $3^{\text {er }}$ Coautor: Anayansi Cristina, Hernández-Ábrego / ORC ID: 0000-0002-8882-4842, CVU CONACYT ID: 748036

\section{Resumen}

En la actualidad, las aplicaciones móviles han incrementado su uso en todos los ámbitos de la vida cotidiana. Entre otras cosas, lo anterior se debe a la facilidad en el intercambio de información. En el ITO se propone una aplicación móvil que complementa la plataforma de Ambiente Virtual para Capacitación del Personal Docente. Dicha aplicación permite la conexión con la plataforma, ingresar al curso donde está inscrito el usuario; y de este modo, el usuario recibe notificaciones de fechas importantes como pueden ser: fechas de entrega de tareas y actividades. Lo anterior es posible, ya que la aplicación se enlaza directamente al calendario de Google. Con estas funciones, como una extensión de la plataforma virtual, facilita al participante de cursos desarrollar las actividades en tiempo y forma. La aplicación se desarrolla basándose en el modelo incremental.

Aplicación móvil, Cursos, Notificaciones

\begin{abstract}
Currently, mobile applications have increased their use in all areas of daily life. Among other things, the above is due to the ease in the exchange of information. The Institute Technologic de Oaxaca proposes a mobile application that complements the Virtual Environment Platform for Professor Training. This application allows the connection with the platform, enter the course where the user is registered; and in this way, the user receives notifications of important dates such as dates of delivery of tasks and activities. The above is possible, since the application links directly to the Google calendar. With these functions, as an extension of the virtual platform, it facilitates the course participant to develop the activities in a timely manner. The application is developed based on incremental model.
\end{abstract}

Mobile app, Courses, Notifications

\footnotetext{
* Correspondencia del Autor (Correo electrónico: evarafaelperez@ gmail.com)

$\dagger$ Investigador contribuyendo como primer autor.
} 


\section{Introducción}

Como sabemos hoy en día, las Tecnologías de la Información y Comunicación (TIC) juegan un rol muy importante en todas las actividades del ser humano, y para la educación como eje rector de la sociedad tambien es fundamental. La educación a distancia basada en las tecnologías y la comunicación brinda a los docentes la posibilidad de capacitación y actualizacion mediante la implementación de los Entornos Virtuales de Aprendizaje (EVA).

El Instituto Tecnológico de Oaxaca (ITO) tiene como objetivo promover el desarrollo profesional de los docentes mediante un acompañamiento en su labor pedagógica, por lo que es importante la capacitación y actualizacion profesional del personal docente; la aplicación móvil para el seguimiento de los cursos es un proyecto que fue realizado y que forma parte del desarrollo integral de la plataforma de Ambiente Virtual para la Capacitación del Personal Docente del InstitutoTecnológico de Oaxaca, que ofrece cursos virtuales de capacitación y actualización de manera permanente.

El desarrollo de la aplicación móvil tiene como objetivo el seguimiento y notificaciones de las actividades asignadas en los cursos, así como el envió de tareas en tiempo y forma a la plataforma de Ambiente Virtual.

Los beneficios que tiene la aplicación móvil es el seguimiento y notificaciones de manera oportuna de los cursos que los docentes estan llevando, cuenta con la función de podcast, que hace posible que el participante pueda retomar la clase que se perdió en el momento que lo requiera durante el periodo del curso; otro beneficio que brinda es en el momento cuando se le asignen las nuevas tareas y actividades del curso desde la plataforma, éstas se enlazan directamente al calendario de Google tomando en cuenta la fecha y la hora, cuenta con la emisión de alertas, así mismo envía las notificaciones de las actividades próximas a realizarse, esto beneficia a los participantes para la entrega de las tareas a tiempo, propiciando así, el aprendizaje individual y significativo por parte del participante.
Con base en lo anterior, el presente trabajo se compone de los siguientes apartados: planteamiento del problema, metodología de software, tipos de usuarios, requerimientos, desarrollo, resultados, conclusiones y referencias bibliográficas.

\section{Planteamiento del problema}

El Instituto Tecnológico de Oaxaca, a través del departamento de desarrollo académico tiene como función principal el mantener al personal docente actualizado, por esta razón dentro de la Institución se ha desarrollado una plataforma virtual para la capacitación docente, que resuelve la problemática que se ha reflejado en los últimos periodos, como es la inasistencia del personal docente a los cursos, debido a que las actividades asignadas a cada uno se superponen con los horarios de los cursos ofertados lo que provoca la falta de participación por parte del personal.

La plataforma Virtual cuenta con los siguientes módulos, personas, usuarios, cursos, reportes, gestión de curso, chat, oferta de cursos, sin embargo, no cuenta con una versión móvil que alerte y notifique las nuevas actividades de los cursos, así como el envió de tareas en tiempo y forma, esto impide al participante poder avanzar con alguna de las actividades asignadas o bien que se retrase en la entrega de las actividades y tareas; no cuenta con una sección que permita retomar algún tema del curso en caso de retrasos de algún participante, por lo que no tiene la posibilidad de recuperar los recursos para su seguimiento.

$\mathrm{Al}$ momento en que el participante sube la tarea a la plataforma virtual, únicamente se despliega un mensaje emergente de confirmación y no envía notificaciones de recibido, por lo que no se tiene la certeza de que fue enviada correctamente; otro inconveniente se presenta cuando el administrador del curso crea una tarea, no se tiene establecido una fecha de entrega, por lo tanto, no se puede tomar en cuenta si ésta fue entregada en tiempo y forma.

Finalmente, otro aspecto que no contempla la plataforma Virtual, es en el momento en que el facilitador califica la tarea, no genera notificaciones para el participante, es decir, no muestra un mensaje donde especifique que la tarea ha sido calificada. 


\section{Metodología}

Para el proyecto, denominado Aplicación móvil para el seguimiento de los cursos de la plataforma de Ambiente Virtual de Aprendizaje, se utilizó la metodología de desarrollo Incremental propuesto por Harlan Mills en 1980, esta metodología combina elementos del modelo lineal Secuencial con la filosofía interactiva de construcción de prototipos, aplica secuencias lineales de forma escalonada mientras progresa el tiempo en el calendario.

Cada secuencia lineal produce un incremento del software, es de naturaleza interactiva brindando al final de cada incremento la entrega de un producto completamente operacional. Las etapas son: análisis, diseño, codificación y pruebas, en este proyecto se realizaron tres incrementos, que fueron desarrollados y evaluados por parte del usuario final.

\section{Tipos de usuarios}

Se identificaron dos tipos de usuario, con sus respectivos roles y privilegios que les permiten el acceso a la aplicación movil:

Usuario Facilitador; tiene acceso a las opciones de gestión, así como ver a los participantes, contenido del curso, tareas, foros, e-rúbricas, evaluaciones $\mathrm{y}$ asignación de calificaciones.

Usuario Participante, es el usuario docente que tiene acceso a la oferta e inscripción de cursos, desarrollo de actividades, subir las tareas, comentarios en foro y ver el seguimiento de cada curso de manera personal.

\section{Desarrollo}

1. Etapa de análisis. En esta etapa se generó el plan de incrementos a realizar durante el desarrollo de la aplicación con base en las necesidades del usuario como se muestra en la tabla 1.

\begin{tabular}{|l|l|l|}
\hline \multicolumn{3}{|c|}{ Plan de Incrementos } \\
\hline $\begin{array}{l}\text { Primer } \\
\text { Incremento }\end{array}$ & $\begin{array}{l}\text { Segundo } \\
\text { Incremento }\end{array}$ & $\begin{array}{l}\text { Tercer } \\
\text { Incremento }\end{array}$ \\
\hline $\begin{array}{l}\text { Visualizar } \\
\text { pantalla de } \\
\text { acceso }\end{array}$ & $\begin{array}{l}\text { Agregar } \\
\text { contenido al } \\
\text { curso }\end{array}$ & $\begin{array}{l}\text { Notificación a los } \\
\text { participantes } \\
\text { cuando se crea } \\
\text { una nueva tarea }\end{array}$ \\
\hline $\begin{array}{l}\text { Visualizar } \\
\text { perfil usuario }\end{array}$ & $\begin{array}{l}\text { Realizar una } \\
\text { tarea }\end{array}$ & $\begin{array}{l}\text { Notificaciones de } \\
\text { recibido al } \\
\text { participante cuando } \\
\text { éste envía su tarea. }\end{array}$ \\
\hline $\begin{array}{l}\text { Inscripción a } \\
\text { cursos }\end{array}$ & $\begin{array}{l}\text { Visualizar } \\
\text { tareas }\end{array}$ & $\begin{array}{l}\text { Asignar calificación } \\
\text { a cada tarea. }\end{array}$ \\
\hline $\begin{array}{l}\text { Agregar fecha de } \\
\text { entrega } \\
\text { al crear una tarea }\end{array}$ & $\begin{array}{l}\text { Crear } \\
\text { un foro }\end{array}$ & $\begin{array}{l}\text { Conexión con API } \\
\text { de Google Calendar }\end{array}$ \\
\hline $\begin{array}{l}\text { Validar que las } \\
\text { tareas sean } \\
\text { enviadas en } \\
\text { tiempo y forma }\end{array}$ & $\begin{array}{l}\text { Responder en } \\
\text { un foro }\end{array}$ & $\begin{array}{l}\text { Notificación a } \\
\text { los participantes } \\
\text { cuando su tarea } \\
\text { enviada ha sido } \\
\text { calificada. }\end{array}$ \\
\hline
\end{tabular}

Tabla 1 Plan de Incrementos

Fuente: Elaboración Propia

Tambien, se crearon las diferentes historias de usuario para determinar los requerimientos del software; una historia de usuario describe la funcionalidad que será valiosa para un usuario o comprador de un sistema o software", (Mike, 2016), es una representación de un requisito escrito en una o dos frases usando el lenguaje común del usuario. Como resultado se obtuvieron los requerimientos funcionales y no funcionales, a manera de ejemplo se muestra en la tabla 2.

\begin{tabular}{|l|l|}
\hline \multicolumn{2}{|c|}{ Historia de Usuario } \\
\hline Número: 3 & Usuario: participante \\
\hline Nombre: Inscripcion al curso \\
\hline Prioridad: Alta & Riesgo: Medio \\
\hline Puntos estimados: 0.5 & Incremento: 2 \\
\hline $\begin{array}{l}\text { Descripción: } \\
\text { Como participante requiero incribirme a los cursos } \\
\text { ofertados }\end{array}$ \\
\hline $\begin{array}{l}\text { Observaciones } \\
\text { Para ingresar a la opcion inscripción de cursos se debe } \\
\text { estar logueado como participante e ingresar a la sección } \\
\text { de oferta de cursos }\end{array}$ \\
\hline
\end{tabular}

Tabla 2 Historia de Usuario: Inscripción del participante Fuente: Elaboración Propia

2. Etapa de diseño. Se realizaron las interfaces de la aplicación móvil, siguiendo las líneas de diseño establecidas por Google para la apariencia de aplicaciones moviles del sistema operativo Android, tomando encuenta el diseño y colores de la plataforma de Ambiente virtual de aprendizaje ya implementada. 
3. Etapa de Codificación. Se utilizó Java con el IDE Android Studio proporcionado por Google como el IDE oficial de desarrollo, utilizando el manejador de base MySql Workbench; los resultados estuvieron en función de los incrementos; los módulos que se codifcaron son: Gestión de acceso a la aplicación, gestión de usuarios, asignación de cursos, tareas, creación de foros, seguimiento de cursos y tareas, gestión del podcast, asignación de fecha $\mathrm{y}$ hora de tareas a Calendar y Notificaciones.

4. Etapa de pruebas. En esta etapa se aplicaron diferentes pruebas, por ejemplo las pruebas de aceptación que se basan en la satisfacción del usuario final; como se muestra en la figura 1 donde valida que el dispositivo móvil tenga acceso a internet, que el servidor esté funcionando correctamente y los datos de usuarios sean correctos.

\section{Ambiente Virtual}
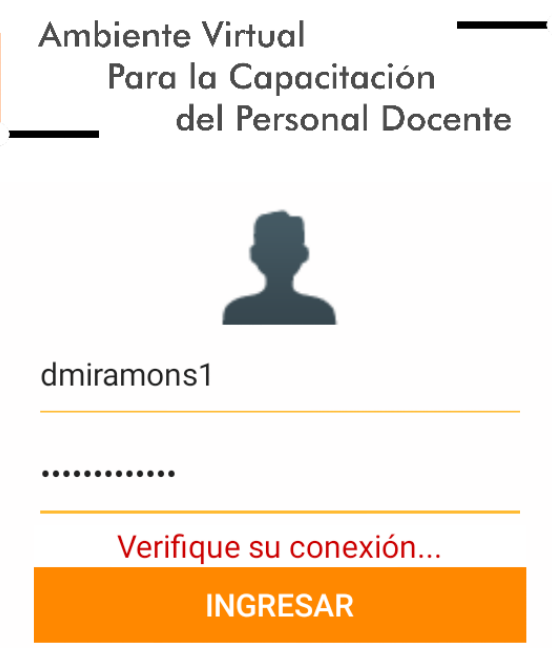

Figura 1 Verificacion de conexión

Fuente: Elaboración Propia

\section{Resultados}

Para la Aplicación móvil se implementaron los siguientes módulos que se muestran dependiendo del rol o roles y privilegios de los usuarios registrados.

\section{Acceso principal a la aplicación Móvil:}

Para poder ingresar es necesario localizar en la pantalla principal del dispositivo móvil el ícono para abrir la aplicación. Se otorgaron los permisos de acceso de acuerdo a los tipos de usuarios, cada usuario cuenta con un nombre de usuario y una contraseña para que pueda ingresar a la aplicación, ver la figura 2.

\section{Ambiente Virtual}

Ambiente Virtual
Para la Capacitación

del Personal Docente

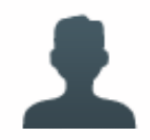

Nombre de usuario

Contraseña

INGRESAR

Figura 2 Pantalla de acceso a la aplicación móvil Fuente: Elaboración Propia

Perfil de usuario. Módulo que permite visualizar la información personal y detalles del usuario como nombre, fecha de nacimiento, clave, cursos y tipo de usuario. Si es usuario participante, muestra los cursos en los que se encuentra inscrito, si es usuario facilitador, muestra los cursos que está impartiendo, ver figura 3 .

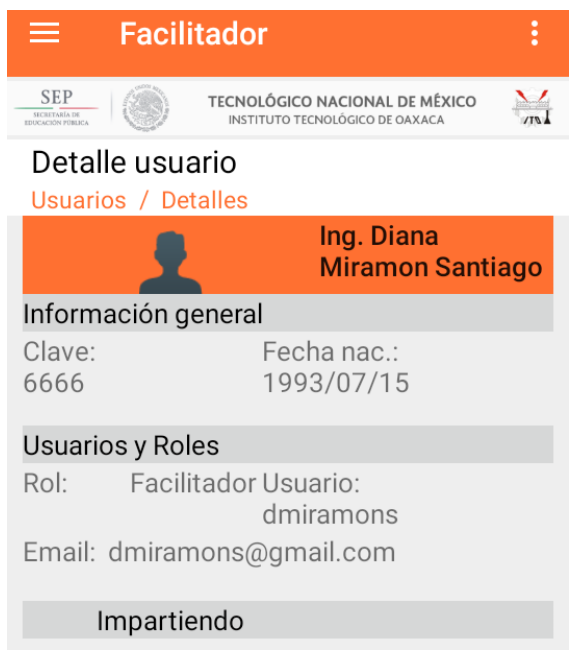

Figura 3 Visualizar información del usuario Fuente: Elaboración Propia 
- Gestión de cursos, este módulo cuenta con dos opciones: Visualización e Inscripción de cursos.

a. Visualización de los cursos, esta opción le permite al usuario de tipo "participante" únicamente ver la información de los cursos ofertados en el momento, el período, las horas de duración y tipo de curso; ver figura 4.

\begin{tabular}{|c|c|c|}
\hline \multicolumn{2}{|c|}{$\equiv \quad$ Participante } & $\vdots$ \\
\hline SEP & 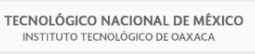 & $\frac{1}{m a t}$ \\
\hline \multicolumn{3}{|c|}{ 玤 } \\
\hline \multicolumn{3}{|l|}{ Curso: } \\
\hline \multicolumn{3}{|c|}{ Programación Lógica y funcional } \\
\hline \multicolumn{3}{|c|}{ Num. registro: SCC-1019 } \\
\hline Inicio: & 2018-02-06 & \\
\hline Terminación: & $2018-03-23$ & \\
\hline \multirow{2}{*}{$\begin{array}{l}\text { Tipo: } \\
\text { Hora: }\end{array}$} & Profesional & \\
\hline & 500 & \\
\hline \multicolumn{3}{|c|}{ Cupo: $\quad 20$} \\
\hline \multicolumn{3}{|c|}{ Min. aprobatorio: 70} \\
\hline \multicolumn{3}{|c|}{ Objetivo: } \\
\hline \multicolumn{3}{|c|}{$\begin{array}{l}\text { Conocer los principios lógicos y funcionale } \\
\text { de la programación para identificarlos y } \\
\text { aplicarlos en la resolución de problemas a } \\
\text { través del diseño de agentes inteligentes. }\end{array}$} \\
\hline \multicolumn{3}{|c|}{ Descripcion } \\
\hline \multicolumn{3}{|c|}{$\begin{array}{l}\text { La asignatura de Programación Lógica y } \\
\text { Funcional aporta al perfil del Ingeniero en } \\
\text { Sistemas Computacionales la capacidad de } \\
\text { desarrollar habilidades para la } \\
\text { generación de soluciones automatizadas } \\
\text { basadas en lenguajes de inteligencia } \\
\text { artificial, considerando el entorno y la } \\
\text { aplicación de diversas técnicas, } \\
\text { herramientas }\end{array}$} \\
\hline
\end{tabular}

Figura 4 Visualización de los cursos del participante Fuente: Elaboración Propia

También le permite al usuario tipo "Facilitador" visualizar los cursos: por módulos, cursos, temas por módulos, ver figura 5.

\begin{tabular}{|c|c|c|c|}
\hline \multicolumn{4}{|c|}{$\equiv \quad$ Facilitador } \\
\hline 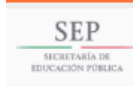 & \multicolumn{2}{|c|}{$\begin{array}{l}\text { TECNOLÓGICO NACIONAL DE MEXICO } \\
\text { INSTITUTO TECNOLÓGICO DE OAXACA }\end{array}$} & $\frac{1}{1001}$ \\
\hline \multicolumn{4}{|c|}{ Cursos impartidos } \\
\hline \multicolumn{2}{|c|}{ Nombre del curso } & Horas & Cupo \\
\hline \multicolumn{2}{|c|}{ Taller de Sistemas Operativos } & 40 & 20 \\
\hline \multicolumn{2}{|c|}{ Tópicos de Programación } & 50 & 20 \\
\hline \multicolumn{2}{|c|}{ Programación Lógica y funcional } & 500 & 20 \\
\hline
\end{tabular}

Figura 5 Visualizar los cursos del Facilidator Fuente: Elaboración Propia

b. La opción de inscripción, le permite la inscripción del usuario al curso deseado, mediante un botón inscribirme o bien desincribirse, para ambos casos, el sistema envía las notificaciones al usuario al correo electrónico y al móvil del usuario. Ver figura 6.

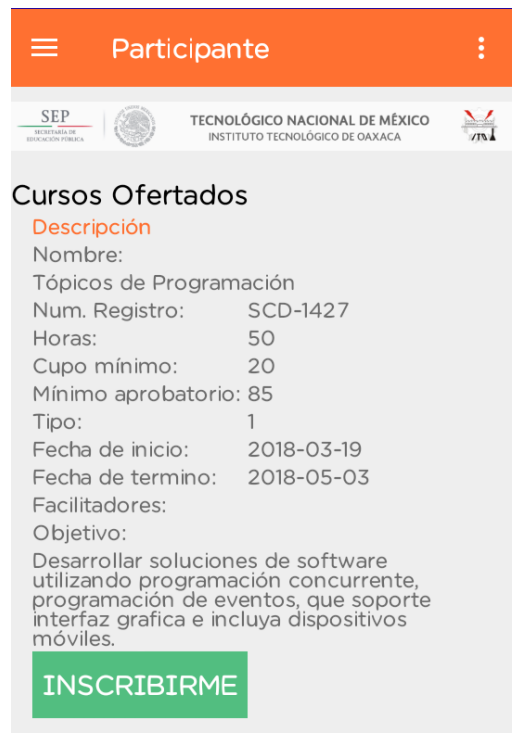

Figura 6 Inscripción al curso Fuente: Elaboración Propia

Gestión de foros, módulo donde el usuario Facilitador crea los foros de discusión para los participantes y realiza comentarios. Ver figura 7.

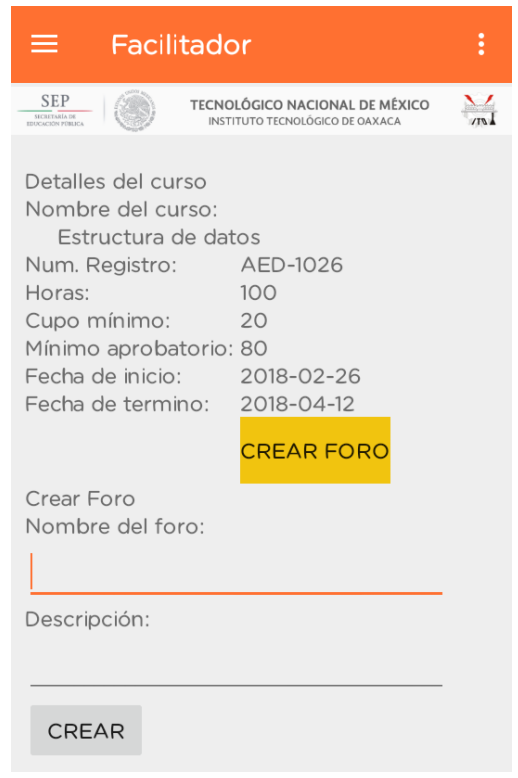

Figura 7 Creación de foros

Fuente: Elaboración Propia

Asi mismo el usuario participante: permite visualizar los foros disponibles si está inscrito en el curso, puede responder o hacer comentarios. 
Gestión de tareas. En este módulo el usuario facilitador: puede revisar $\mathrm{y}$ asignar una calificación a las tareas y actividades después que el participante haya enviado su tarea. Por el lado del Participante puede consultar si hay tareas pendientes, sí la actividad requiere cargar y enviar algún archivo, este debe ser igual o menor a $5 \mathrm{MB}$, visualizar el progreso/estado y detalles de las tareas o actividades que han sido enviadas: fecha de entrega, calificación, entre otros, ver figura 8 .

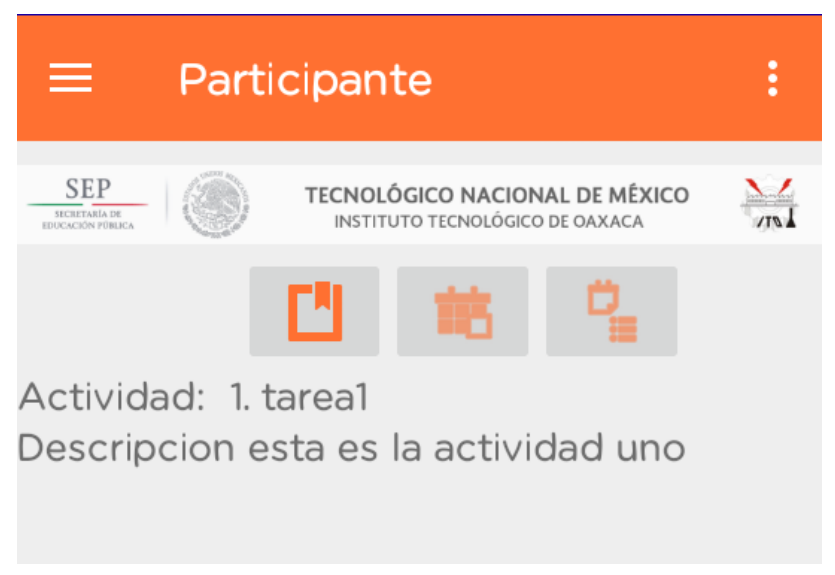

Figura 8 Gestión de tareas del participante

Fuente: Elaboración Propia

\section{Conclusiones}

Con el uso de las Tecnologías de la Información y Comunicación en la educación ha revolucionado la manera de aprender e interectuar mediante los ambientes virtuales de aprendizaje, permite que los participantes puedan compartir experiencias, trabajar en equipo colaborativo, aprender a su ritmo y adquirir nuevos conocimientos.

La implementación de la Aplicación móvil forma parte fundamental del proyecto integrador de la plataforma de Ambiente Virtual de Aprendizaje para la capacitación del personal docente del Tecnológico de Oaxaca, cuyo objetivo es el seguimiento y notificaciones de las actividades asignadas en los cursos, así como el envió de tareas a la plataforma de ambiente virtual. Los beneficios que tiene la aplicación móvil es el seguimiento y notificaciones de manera oportuna de los cursos que los docentes están llevando; ya que permite notificar al usuario participante cuando se ha creado una nueva tarea, cuando la tarea ha sido enviada, comentada y calificada.
Además funciona como recordatorio cuando el tiempo de entrega de una tarea esté a punto de vencer; cuenta con la función de podcast, que hace posible que el participante pueda retomar la clase que se perdió en el momento que el usuario lo requiera durante el periodo del curso mediante el acceso a multimedia desde la aplicación; cuando se le asignen las nuevas tareas y actividades del curso desde la plataforma virtual, éstas se enlazan directamente al calendario de Google tomando en cuenta la fecha y la hora; cuenta con la emisión de alertas, así mismo envía las notificaciones de las actividades próximas a realizarse, esto beneficia a los participantes para la entrega de las tareas a tiempo; la aplicación móvil también busca establecer la comunicación entre el facilitador y el participante durante el curso que esté llevando.

Finalmente concluyo que los ambientes virtuales fortalecen el aprendizaje colaborativo y con la interación de las aplicaciones móviles permiten que el docente aprenda en cualquier momento, para que pueda ser aplicado en el aula en beneficio de los estudiantes de la Institución.

\section{Referencias}

Adell, J.; Castell, J. \& Pascual, J., (2004), "Selección de un entorno virtual de enseñanza/aprendizaje de código fuente abierto para la Universidad Jaume I", [en línea], Disponible en http://cent.uji.es

Álvarez Miguel A. (Mayo de 2001). Desarrollo Web. Recuperado de http://www.desarrolloweb.com/articulos/392.ph p Clarenc, C. A.; S. M. Castro, C. López de Lenz, M. E. Moreno \& N. B. Tosco (Diciembre, 2013). Analizamos 19 plataformas de eLearning: Investigación colaborativa sobre LMS. Grupo GEIPITE, Congreso Virtual Mundial de eLearning. Recuperado de http://goo.gl/sVEmg6

Cobo, Ángel y Gómez. 2005. PHP y MySQLtecnologías para el desarrollo de aplicaciones web. S.1.: Ediciones Díaz de Santos, 2005. Recuperado de https://www.ecured.cu/Sistema_Gestor_de_Bas e_de_Datos

De miguel Adoración., Piattini Mario., Marcos Esperanza. (2000). Diseño de base de datos relacionales. Alfaomega Eguíluz Pérez Javier. (Junio de 2008). Introducción a JavaScript. Recuperado de https://goo.gl/y0cWEG

RAFAEL-PÉREZ, Eva, MORALES-HERNÁNDEZ, Maricela, MIRAMÓNSANTIAGO, Diana del Carmen y HERNÁNDEZ-ÁBREGO, Anayansi Cristina. Aplicación móvil para el seguimiento de los cursos de la plataforma de Ambiente Virtual de Aprendizaje. Revista de Tecnologías de la Información y Comunicaciones. 2019 
Eguíluz Pérez Javier. (Junio de 2008). Introducción a JavaScript. Recuperado de https://goo.gl/y0cWEG

Facultad militar nueva granada (s.f). Ambientes virtuales. Recuperado de http://www.umng.edu.co/ambientes-virtuales J. Gutiérrez Javier (s.f). ¿Qué es un framework web? Recuperado de https://goo.gl/wqmUyB

IBM (2012), El desarrollo de aplicaciones móviles nativas, web o hibridas, Consultado el 28 de noviembre de 2018, Disponible: ftp://ftp.software.ibm.com/la/documents/gb/co mmons/27754_IBM_WP_Native_Web_or_hyb rid_2846853.pdf

Lafosse, J. (s.f.). Struts 2: El framework de desarrolo de aplicaciones Java EE 2014. Cornellà de Llobregat (Barcelona): ENI.

Merino (2014), ¿Qué es un API y para qué sirve?, Consultado el 2 de diciembre de 2018 Disponible:

https://www.ticbeat.com/tecnologias/que-esuna-api-para-que-sirve/

Nevado, M. (2010). Introducción a Las Bases de Datos Relacionales. España: Visión Libros

Otwell Taylor (s.f). Documentation. Recuperado de https://laravel.com/docs/5.3/ Pressman, R., \& Murrieta, J. (2006). Ingeniería del software un enfoque practico ( $6^{\mathrm{a}}$ Edición ed.). McGraw-Hill.

Pressman, R., \& Murrieta, J. (2006). Ingeniería del software un enfoque práctico $\left(6^{\mathrm{a}}\right.$ Edición ed.). McGraw-Hill.

Sommerville, Ian M. I. (2005). Ingeniería del software. Pearson Educación.

Zaefferer Jörn. (s.f). Validación de formularios con jQuery. Recuperado de https://Jqueryvalidation.org/ 